\title{
Versatile peroxidases: super peroxidases with potential biotechnological applications-a mini review
}

\begin{abstract}
Ligninolytic enzymes play a crucial role in the carbon cycle on account of their ability to degrade the macromolecule lignin. Versatile peroxidase is a high redox potential enzyme of the lignin degrading family of enzymes widely studied for its unique characteristics of degradation of aromatics without use of redox mediators and presence of polyvalent catalytic sites. These traits make them useful in diverse biotechnological and industrial applications which include textile bleaching, production of bioethanol, bioremediation of xenobiotic compounds, degradation of EDCs and in enhancing the digestibility of animal feed. In this article the features of versatile peroxidases and their potential industrial and biotechnological applications are highlighted.
\end{abstract}

Keywords: lignolytic enzyme, white rot fungi, lignin degradation, ruminant nutrition
Volume 4 Issue 2 - 2016

\author{
Aarthi Ravichandran, Manpal Sridhar \\ National Institute of Animal Nutrition and Physiology, India \\ Correspondence: Manpal Sridhar, Bioenergetics \& \\ Environmental Sciences Division, National Institute of Animal \\ Nutrition and Physiology, Adugodi, Bengaluru 560030, Karnataka, \\ India, Tel +9| $80257 \mid$ | 304,094 4895 0222, Fax +9| 80257 I \\ 1420, Email manpalsridhar@yahoo.co.uk
}

Received: October 03, 2016 | Published: November 23, 2016

\section{Introduction}

Versatile peroxidases are enzymes belonging to the super family of plant, fungal and bacterial peroxidases specifically, the class II subfamily, the major rationale of which seems to be degradation of lignin in wood. The heme peroxidases of the class II family, Lignin peroxidase, Manganese peroxidase and Versatile peroxidase operate through a central heme protoporphyrin using hydrogen peroxide as the electron acceptor. The enzyme versatile peroxidase as its name signifies exhibit a versatile nature by oxidizing diverse substrates under different environmental conditions. ${ }^{1}$ Interestingly, Versatile peroxidases possess the catalytic features of Lignin peroxidase, Manganese peroxidase and other microbial peroxidases in degradation of aromatics, attesting it as a hybrid peroxidase. ${ }^{2}$ In aid to this fact, versatile peroxidase harbours multiple active sites for oxidation of $\mathrm{Mn}^{2+}$, low and high molecular weight substrates. ${ }^{2}$ These proteins are monomeric glycoproteins with a molecular weight of approximately 40-50 KDa with four conserved disulfide bridges and two conserved calcium binding sites, a signature of class II family of plant, fungal and bacterial peroxidases. The attractive feature of these enzymes is the substrate oxidation without requirement of redox mediators unlike Manganese peroxidase whose obligatory co- substrate is manganese and Lignin peroxidase which oxidizes the substrate through redox mediators like veratryl alcohol. ${ }^{3}$ Versatile peroxidases catalyze oxidation of wide range of substrates from hydroquinones, substituted phenols to bulky recalcitrant lignin directly, without redox mediators. Their $\mathrm{pH}$ optimum for oxidation of aromatics varies depending on the presence of manganese in the medium; oxidation of $\mathrm{Mn}^{2+}$ occurs at an optimum of $\mathrm{pH} 5.0$ in contrast to the acidic $\mathrm{pH}(\mathrm{pH} 3.0)$ in the absence of manganese. These optimum $\mathrm{pH}$ values correspond to the $\mathrm{pH}$ optima of Manganese peroxidase and Lignin peroxidase oxidation ${ }^{3}$. Versatile peroxidases have been characterized in Pleurotus and Bjerkandera species and are reported to be present in Panus, Calocybe, Trametes, Lepista, Dichomitous and Spongipellis. ${ }^{4}$ The distinctive feature of this class of enzymes in oxidation of diverse array of substrates without prerequisite of redox mediators presents it as an attractive choice for multitude of biotechnological and industrial applications.

\section{Production of versatile peroxidase}

White rot fungi are a group of wood rotting fungi recognized for their ability of degradation of lignin resulting in bleaching of wood exposing the cellulose and hemicellulose fibres. These are the only organisms known to completely degrade lignin polymer to carbon dioxide and water. ${ }^{5}$ White rot fungi encompass majorly basidiomycetes phyla which accomplish degradation of wood through simultaneous or selective delignification. Selective degradation of wood by certain basidiomycetes is preferred as its activity focuses on macromolecular lignin leaving behind the energy rich celluloses and hemicelluloses for constructive exploitation. ${ }^{5}$ These white rot fungi are robust organisms which perform oxidative lignin breakdown through its battery of ligninolytic enzymes. The ligninolytic enzyme system is exclusive for white rot fungi comprising of phenol oxidase like laccase, heme peroxidases like Lignin peroxidase, Manganese peroxidase, Versatile peroxidase and $\mathrm{H}_{2} \mathrm{O}_{2}$ producing enzymes like aryl alcohol oxygenases and dehydrogenases. Although there are reports of laccase expression in higher plants, bacteria, arthropods, insects and yeasts, heme peroxidases especially Versatile peroxidase are exclusively expressed in white rot fungi. These enzymes are known to be secreted by white rot fungi during their secondary metabolic phase. ${ }^{6}$

Submerged and solid state modes of fermentation are employed for the production of Versatile peroxidase from these fungi. Solid state fermentation makes use of natural substrates like rice straw, wheat straw with reduced water activity for production of enzymes. Solid state fermentation exhibits tremendous advantage for production of these enzymes as they mimic the natural substrate conditions of the fungi. Significant amount of Versatile peroxidase have been produced by solid state fermentation of Pleurotus eryngii and Pleurotus Ostreatus on wheat straw, Saw dust, banana peel. ${ }^{7,8}$ Disparately, submerged fermentation involves culturing of microorganisms in free flowing liquid substrates with high water activity. Some examples of Versatile peroxidase produced under submerged fermentation are growth of Pleurotus ostreatus, Bjerkandera in glucose-peptone broth and glucose ammonium medium. ${ }^{7}$ Additionally, immobilization of cells offers significant advantages over submerged cell suspension. Immobilization of fungal cells has been reported for production of laccase wherein immobilization methods are stated to improve the stability and half life of laccases enabling efficient biotechnological application of this enzyme. ${ }^{9}$ However, there is a dearth of reports of Versatile peroxidase production under immobilized conditions. 


\section{Mechanism of action}

Versatile peroxidases are unique among the heme peroxidases for its polyvalent catalytic sites for oxidation of $\mathrm{Mn}^{2+}$, low and high molecular weight substrates. ${ }^{10}$ The catalytic cycle of oxidation of low molecular substrates at the heme center is initiated by binding of hydrogen peroxide to the ferric state of heme forming an iron peroxide complex. This activated heme complex is a two electron deficient intermediate designated as compound I, a radical with significant oxidizing ability. Compound I is reduced to compound II, a one electron deficient compound and finally to resting form of enzyme with concomitant oxidation of two substrates..$^{10}$ While the initial reaction of compound I formation is $\mathrm{pH}$ independent, the latter reduction steps are $\mathrm{pH}$ dependent with the oxidized substrates yielding radical products which undergo several non-enzymatic reactions based on the nature of the substrate to yield final products. The nonenzymatic reactions include aromatic ring cleavage, hydroxylation, demethoxylation, ether bond cleavage, side chain cleavage and phenol formation. ${ }^{10}$ The reaction scheme for oxidation of aromatic substrates is described in Figure 1.

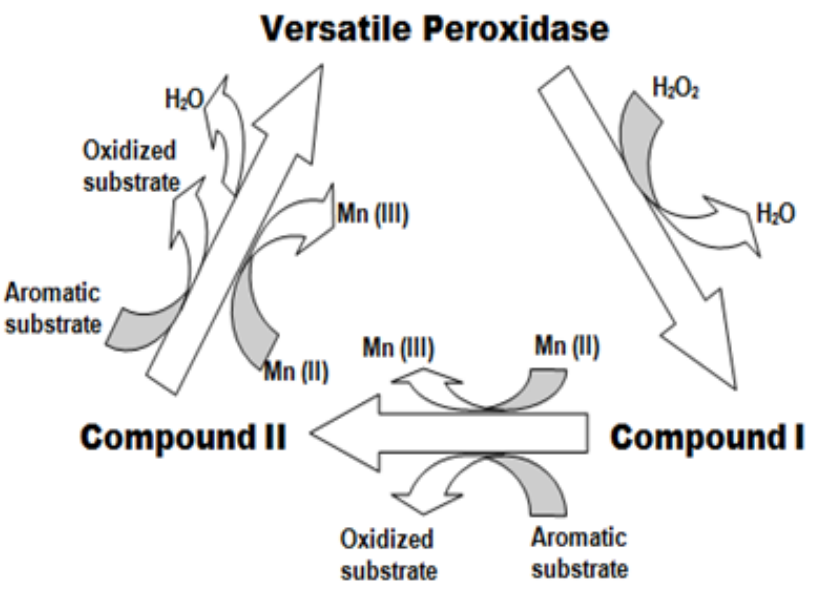

Figure I The schematic representation of the oxidation of aromatic substrates and manganese by versatile peroxidases."

Interestingly, Versatile peroxidase possesses an additional manganese oxidation site besides the main heme channel utilized for substrate oxidation. Manganese requisite for oxidation by the enzyme is available in considerable quantities in wood and also in soil. This manganese is oxidized by the enzyme in the narrow access channel close to heme propionate, where it chelates with the organic acids secreted by the fungi like glyoxalate, oxalate and lactate to form a $\mathrm{Mn}^{3+}$ organic acid complex which acts a diffusing oxidizer in oxidation of aromatic substrates. ${ }^{11}$ This property makes this enzyme analogous to Manganese peroxidase, a ligninolytic enzyme which functions through manganese oxidation. A note worthy feature of Versatile peroxidase which puts it similar to Lignin peroxidase is the high molecular weight substrate oxidation through Long Range Electron Transfer (LRET) initiated at a surface exposed tryptophan residue of the enzyme. ${ }^{11}$ The tryptophan radical is supposed to exist in neutral form in an acidic environment favourable for substrate binding. Electrons are transferred from tryptophan to redox cofactor heme of the enzyme where electron abstraction from the substrate causes cleavage of intramolecular bonds through non-enzymatic reactions. ${ }^{11}$ Conclusively, versatile peroxidase is a hybrid peroxidase with structural and functional features related to two other members of ligninolytic heme peroxidases viz. Lignin peroxidase and Manganese peroxidase. Genetic architecture also confirms this concept where in the genetic elements of versatile peroxidase are shown to possess significant similarity to Lignin peroxidase and Manganese peroxidase genes. In Phanerochaete chrysosporium, the most extensively studied white rot fungus, the Lignin peroxidase gene is reported to exhibit high relativity to Versatile peroxidase of Bjerkandera than its Manganese peroxidase gene. ${ }^{12}$

\section{Structural characteristics}

As elucidated earlier, versatile peroxidase is a glycosylated heme protein with two solvent access channels; the wider channel for access by hydrogen peroxide and narrow access channel for access to manganese. The wider access channel promotes contact between hydrogen peroxide and the heme cofactor where the activated oxo-ferryl intermediate binds the substrate molecules. ${ }^{13}$ The heme propionate is stabilized by two helices with $\mathrm{Ca}^{2+}$ and two histidine residues one at proximity to heme and another at the distal end. The manganese cation binding site is stabilized by three acidic residues specifically tri-carboxylates of glutamate and aspartate residues. ${ }^{12}$ The heme channel and tri-carboxylate manganese binding site of this peroxidase are illustrated in Figure 2. High redox potential substrate oxidizing property of versatile peroxidase is rendered by its highly catalytic tryptophan neutral radical, an intermediate generated by heme reaction with $\mathrm{H}_{2} \mathrm{O}_{2}{ }^{14}$

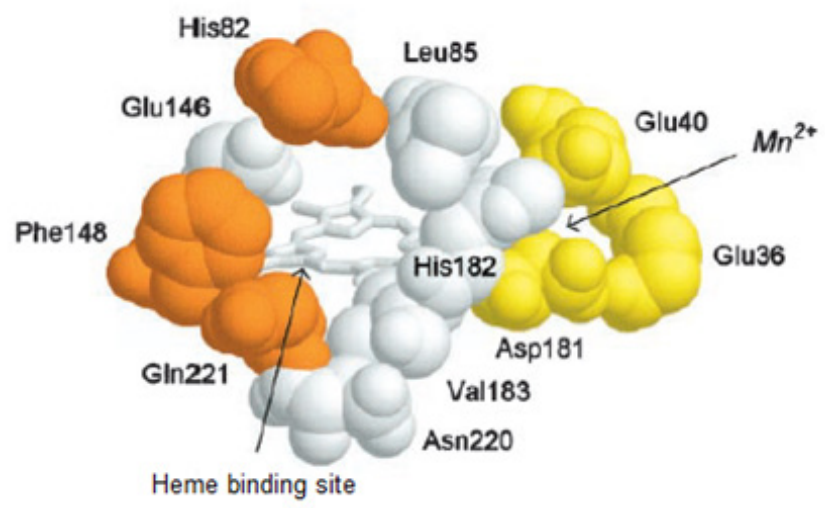

Figure 2 Heme opening channel with the stabilizing amino acid residues in the versatile peroxidase of Pleurotus eryngii. ${ }^{15}$ Manganese oxidation site is highlighted in yellow with symbolic representations of aspartate and glutamate residues.

\section{Biotechnological and industrial applications of versa- tile peroxidase}

The presence of diverse catalytic sites, non-requisition of redox mediators and ability to oxidize a wide range of substrates spanning from low to high redox potentials proposes versatile peroxidase as the most widely preferred and attractive choice for biotechnological applications among the basidiomycetes peroxidases.

\section{Bio-remediation}

Textile dyeing and related industrial applications use a wide spectrum of synthetic dyes which are released in the waste water effluents. These dyes are highly resistant to microbial attack and also least treated by conventional methods. It has been reported that versatile peroxidase can efficiently oxidize high redox potential dyes like Reactive Black 5, Reactive Blue 19 and also substituted phenols, 
hydroquinones. ${ }^{15}$ Pleurotus ostreatus Versatile Peroxidase is reported to decolourize structurally different group of dyes triarylmethane, heterocyclic azo, anthraquinone and polymeric dyes. ${ }^{16}$ On the same lines, the enzyme from Bjerkandera fumosa is stated to decolorize azo and anthraquinone dyes in both manganese dependent and independent reactions. ${ }^{17} \mathrm{Further}$, these fungi are also able to degrade the xenobiotic aromatic pollutants leached into the terrestrial and aquatic ecosystems through the use of herbicides and pesticides. ${ }^{18}$ Pleurotus ostreatus, Pleurotus eryngii, Pleurotus pulmonarius and Pleurotus sajor-caju are shown to possess the capability of catalysing the oxidation of chlorinated biphenyls and poly hydroxyl hydrocarbons. ${ }^{18}$

\section{Paper and pulp industry}

Lignocelluloses are the primary raw material for paper pulp industry where pulp bleaching is done to remove the lignin from the fibres. Presence of lignin causes yellowing of paper with age upon its oxidation. Hence fibre materials with reduced kappa number, an indicator of residual lignin content are desired. Chemical methods of delignification pose severe environmental hazards as most of these paper and pulp industries are located close to water bodies due to their requirement of large quantities of water. Ligninolytic enzymes are ecofriendly means of delignification of these lignocellulosic fibres. Pulp bleaching using manganese substituted polyoxometalates assisted by Versatile peroxidase is reported be effective with good reduction in kappa number. ${ }^{19}$

\section{Ruminant nutrition}

Lignocelluloses are an attractive choice for ruminant nutrition given its abundance and the ability of the animal to digest the polysaccharides. Though these lignocellulosic fibres are typically used in feeding animals, they are generally categorized as low quality roughage and often supplemented with concentrates. This is due to the presence of amorphous polymer lignin bonded to the cellulose and hemicellulose fibrils preventing its efficient depolymerisation. Delignification by fungi especially white rot is preferred for their selective and efficient degradation of lignin. Delignification besides exposing the cellulose and hemicellulose macrofibrils, improves the digestibility and nutritive value of the feed. ${ }^{20}$ This is evident from the report on enhancement of ruminant nutrition through the use of fungal pre-treated crop residues. ${ }^{21}$ The delignification process is a complex process of enzymatic combustion as a result of the integrative action of phenol oxidases and heme peroxidases. Reports have demonstrated the effectiveness of Laccase, Manganese peroxidase and Lignin peroxidase in improving the digestibility and nutritive value of crop residues; ${ }^{22}$ however more insight is needed to study the effectiveness of Versatile peroxidase towards this end.

\section{Biofuel production}

Production of second generation biofuel especially from lignocelluloses has received considerable attention in recent years due to its large scale availability and high energy content. The presence of lignin in the plant cell walls negatively impacts the conversion of biomass to biofuel. The conversion of biomass to biofuel is accomplished by fermentation of sugars preceded by pre-treatment and hydrolysis. ${ }^{22}$ Pre-treatment of biomass is required for delignification and hence effective fermentation of cellulose. Biological methods of pre-treatment are efficient compared to physical and chemical methods. Further, biological pre-treatment by fungi besides removing the lignin also reduces the crystallinity of cellulose thereby increasing its porosity. ${ }^{23}$ Versatile peroxidases seem to be attractive solution for pre-treatment of biofuels as they efficiently deconstruct the lignocellulosic polymers without the requirement of redox mediators. The high redox potential of this class of heme peroxidases and ability to act independent of redox mediators are of great significance in their utilization for biofuel production.

\section{Biomedical applications}

Endocrine Disrupting Chemicals(EDCs) are group of anthropogenic or synthetic chemical compounds present in the environment causing adverse effects to the health and behaviour of animals and also other living organisms by altering the functions of the endocrine system. These chemicals include bisphenol, triclosan etc. which are harmful even at trace amounts. The conventional methods of treatment are not effective in complete removal of these EDCs from the environment. ${ }^{24}$ Recently, the capability of versatile peroxidase to degrade the EDCs and reduce their activity was demonstrated. Versatile peroxidase is highly preferred in disruption of these chemicals owing to their high redox potential and independence of redox mediators. Two stage enzymatic system for removal of EDCs at high and at environmental concentrations was studied and reported to be effective. ${ }^{24}$

\section{Textile industry}

Peroxidases are used for dye bleaching in textile industries. Versatile peroxidases hold high potential for these applications as they are effective in removal of diverse synthetic dyes. An alkaline stable versatile peroxidase from Bjerkandera adusta is reported to degrade $\beta$-carotene efficiently making it a widely desired enzyme in de-staining of fabrics in textile industries. ${ }^{25}$ Degradation of complex aromatic structures of most of the synthetic dyes possessing wide spectrum of chemical groups are achieved without the use of redox mediators and to high efficiency. ${ }^{15,26,27}$

\section{Conclusion}

Versatile peroxidases possess unique features among the lignolytic peroxidases suitable for exploitation in diverse applications. The major limitation to the efficient industrial application of this enzyme is the difference in its availability to demand. Efforts on developing an efficient heterologous expression system for this enzyme will be of great benefit for commercial economic utilization of the enzyme.

\section{Acknowledgements}

None.

\section{Conflict of interest}

Author declares that there is no conflict of interest.

\section{References}

1. D Knop, O Yarden, Y Hadar. The ligninolytic peroxidases in the genus Pleurotus: divergence in activities, expression, and potential applications. Applied Microbiology and Biotechnology. 2015;99:1025-1038.

2. AT Martínez, M Speranza, FJ Ruiz-Dueñas, et al. Biodegradation of lignocellulosics: microbial, chemical, and enzymatic aspects of the fungal attack of lignin. Int Microbiol. 2005;8(3):195-204.

3. PR Moreira, E Almeida-Varaa, FX Malcatab, et al. Lignin transformation by a versatile peroxidase from a novel Bjerkandera sp. Strain. Int Biodeterior Biodegrad. 2007;59(3):234-238. 
4. FJ Ruiz-Dueñas, M Morales, MJ Mate, et al. Site-directed mutagenesis of the catalytic tryptophan environment in Pleurotus eryngii versatile peroxidase Biochemistry. 2008;47(6):1685-1695.

5. TK Kirk, RL Farrell. Enzymatic “combustion": the microbial degradation of lignin. Annu Rev Microbiol. 1987;41:465-505.

6. C Palma, L Lloret, L Sepúlveda, et al. Production of versatile peroxidase from Pleurotus eryngii by solid-state fermentation using agricultural residues and evaluation of its catalytic properties. Prep Biochem Biotechnol. 2016;46(2):200-207.

7. L Caramelo, MJ Martínez, ÁT Martínez. A search for ligninolytic peroxidases in the fungus pleurotus eryngii involving alpha-ketogamma-thiomethylbutyric acid and lignin model dimers. Appl Environ Microbiol. 1999;65(3):916-922.

8. Giardina P, Palmieri G, Fontanella B, et al. Manganese peroxidase isoenzymes produced by Pleurotus ostreatus grown on wood sawdust. Arch Biochem Biophys. 2000;376(1):171-179.

9. Guzik U, Hupert-Kocurek K, Wojcieszyńska D. Immobilization as a strategy for improving enzyme properties-application to oxidoreductases. Molecules. 2014;19(7):8995-9018.

10. Busse N, Wagner D, Kraume M, et al. Reaction Kinetics of Versatile Peroxidase for the Degradation of Lignin Compounds. American Journal of Biochemistry and Biotechnology. 2013;9(4):365-394.

11. Ruiz-Dueñas FJ, Martínez ÁT. Microbial degradation of lignin: how a bulky recalcitrant polymer is efficiently recycled in nature and how we can take advantage of this. Microb Biotechnol. 2009;2(2):164-177.

12. Moreira PR, Duez C, Dehareng D, et al. Molecular characterisation of a versatile peroxidase from a Bjerkandera strain. J Biotechnol. 2005;118(4):339-352.

13. Perez-Boada M, Ruiz-Duenas FJ, Pogni R, et al. Versatile peroxidase oxidation of high redox potential aromatic compounds: site-directed mutagenesis, spectroscopic and crystallographic investigation of three long-range electron transfer pathways. J Mol Biol. 2005;354(2):385-402.

14. Pogni R, Baratto MC, Teutloff $\mathrm{C}$, et al. A tryptophan neutral radical in the oxidized state of versatile peroxidase from Pleurotus eryngii: a combined multifrequency EPR and density functional theory study. $J$ Biol Chem. 2006;281(14):9517-9526.

15. Heinfling A, Martínez MJ, Martínez AT, et al. Transformation of industrial dyes by manganese peroxidases from Bjerkandera adusta and Pleurotus eryngii in a manganese-independent reaction. Appl Environ Microbiol. 1998;64(8):2788-2793.
16. Bansal N, Kanwar SS. Peroxidase(s) in environment protection. Sci World J. 2013;2013:714639.

17. Jarosz-Wilkołazka A, Luterek J, Olszewska A. Catalytic activity of versatile peroxidase from Bjerkandera fumosa at different $\mathrm{pH}$. Biocatalysis and Biotransformation. 2008;26(4):280-287.

18. Rodríguez E, Nuero O, Guillén F, et al. Degradation of phenolic and non-phenolic aromatic pollutants by four Pleurotus species: the role of laccase and versatile peroxidase. Soil Biology and Biochemistry. 2004;36(6):909-916.

19. Marques G, Gamelas JA, Ruiz-Dueñas FJ, et al. Delignification of eucalypt kraft pulp with manganese-substituted polyoxometalate assisted by fungal versatile peroxidase. Bioresour Technol. 2010;101(15):5935-5940.

20. Manpal S, Raghavendra B, Dhali A, et al. In vitro evaluation of the effect of exogenous lignolytic enzymes on the nutritive value of Eleusine coracana (ragi straw). Advances in Applied Research. 2014;6(1):45-52.

21. Van Kuijk SJ, Sonnenberg AS, Baars JJ, et al. Fungal treated lignocellulosic biomass as ruminant feed ingredient: a review. Biotechnol Adv. 2015;33(1):191-202.

22. Plácido J, Capareda S. Ligninolytic enzymes: a biotechnological alternative for bioethanol production. Bioresources and Bioprocessing. $2015 ; 2: 23$.

23. Isroi R, Millati S, Syamsiah C, et al. Biological Pretreatment of Lignocelluloses with White-Rot Fungi and its applications: A Review. Bioresources. 2011;6(4):5224-5259.

24. Taboada-Puig R, Lu-Chau TA, Eibes G, et al. Continuous removal of endocrine disruptors by versatile peroxidase using a two-stage system. Biotechnol Prog. 2015;31(4):908-916.

25. D Linke, R Leonhardt, $\mathrm{N}$ Eisele, et al. Carotene-degrading activities from Bjerkandera adusta possess an application in detergent industries. Bioprocess and Biosystems Engineering. 2015;38(6):1191-1199.

26. Ruiz-Duenas FJ, Martinez MJ, Martinez AT. Molecular characterization of a novel peroxidase isolated from the ligninolytic fungus Pleurotus eryngii. Molecular Microbiology. 1999;31(1):223-235.

27. Camarero S, Sarkar S, Ruiz-Dueñas FJ, et al. Description of a versatile peroxidase involved in the natural degradation of lignin that has both manganese peroxidase and lignin peroxidase substrate interaction sites. J Biol Chem. 1999;274(15):10324-10330. 